\title{
Algunas implicaciones didácticas derivadas de la complejidad de los objetos matemáticos cuando estos se aplican a distintos contextos
}

\section{RESUMO}

Vicenç Font

$\frac{\text { vfont@ub.edu }}{\text { 0000-0003-1405-0458 }}$

Universitat de Barcelona, España.

\section{Adriana Breda}

adriana.breda@gmail.com

Universidad de Los Lagos, Chile.

María José Secke

miseckel@ucm.cl

-746X

Universidad Católica de Maule, Chile
Este trabajo es, fundamentalmente, una reflexión teórica. En la primera parte se explica la noción de idoneidad didáctica y, en particular, el criterio de idoneidad epistémica, siendo uno de sus indicadores la "muestra representativa de la complejidad del objeto matemático". En la segunda parte se justifica la presencia de este indicador a partir de una reflexión, realizada desde una perspectiva pragmatista, sobre su relación con la noción de proceso de conexión y de contexto. Se finaliza con una reflexión sobre el uso de dicho descriptor en la formación de profesores de matemáticas.

PALAVRAS-CHAVE: Idoneidad epistémica. Conexión. Contexto. Formación de profesores de matemáticas. 
La noción de idoneidad didáctica propuesta por el Enfoque Ontosemiótico de la Cognición e Instrucción Matemáticos (EOS, a partir de ahora) (GODINO, BATANERO y FONT, 2007; 2008) es una respuesta parcial a la siguiente problemática: ¿Qué criterios se deben utilizar para diseñar una secuencia de tareas, que permitan evaluar y desarrollar la competencia matemática de los alumnos y qué cambios se deben realizar en su rediseño para mejorar el desarrollo de esta competencia? Los criterios de idoneidad pueden servir primero para guiar los procesos de enseñanza y aprendizaje de las matemáticas y, segundo, para valorar sus implementaciones. Los criterios de idoneidad son útiles en dos momentos de los procesos de instrucción. A priori, los criterios de idoneidad son principios que orientan "cómo se deben hacer las cosas". A posteriori, los criterios sirven para valorar el proceso de instrucción efectivamente implementado. En el EOS se consideran los siguientes criterios de idoneidad didáctica (FONT, PLANAS y GODINO, 2010):

$\checkmark \quad$ Idoneidad Epistémica, para valorar si las matemáticas que están siendo enseñadas son "buenas matemáticas".

$\checkmark$ Idoneidad Cognitiva, para valorar, antes de iniciar el proceso de instrucción, si lo que se quiere enseñar está a una distancia razonable de aquello que los alumnos saben, y después del proceso, si los aprendizajes adquiridos están cerca de aquello que se pretendía enseñar.

Idoneidad Interaccional, para valorar si las interacciones resuelven dudas y dificultades de los alumnos.

$\checkmark$ Idoneidad Mediacional, para valorar la adecuación de los recursos materiales y temporales utilizados en el proceso de instrucción.

Idoneidad Emocional, para valorar la implicación (intereses, motivaciones, ...) de los alumnos durante el proceso de instrucción. 
de los criterios. Por ejemplo, todos concordamos que es necesario implementar unas "buenas" matemáticas, pero podemos entender cosas muy diferentes por ello. Para algunos criterios, los descriptores son relativamente fáciles de consensuar (por ejemplo, para el criterio de idoneidad de medios), para otros, como es el caso de la idoneidad epistémica es más difícil. En Breda y Lima (2016), Seckel (2016) y Breda, Pino-Fan y Font (2017) se aporta un sistema de componentes e indicadores que sirve de guía de análisis y valoración de la idoneidad didáctica, que está pensado para un proceso de instrucción en cualquier etapa educativa. A continuación, por cuestiones de espacio, solo se reproducen los componentes e indicadores del criterio de idoneidad epistémica (BREDA y LIMA, 2016, p. 80):

Tabla 1 - Componentes y descriptores de la idoneidad epistémica.

Componentes

\section{Descriptores}

\begin{tabular}{|c|c|c|}
\hline Errores & $\checkmark$ & $\begin{array}{l}\text { No se observan prácticas que se consideren incorrectas desde el } \\
\text { punto de vista matemático. }\end{array}$ \\
\hline Ambigüedades & $\checkmark$ & $\begin{array}{l}\text { No se observan ambigüedades que puedan llevar a la confusión a } \\
\text { los alumnos: definiciones y procedimientos clara y correctamente } \\
\text { enunciados, adaptados al nivel educativo al que se dirigen; } \\
\text { adecuación de las explicaciones, comprobaciones, demostraciones } \\
\text { al nivel educativo a que se dirigen, uso controlado de metáforas, } \\
\text { etc. }\end{array}$ \\
\hline Riqueza de procesos & $\checkmark$ & $\begin{array}{l}\text { La secuencia de tareas contempla la realización de procesos } \\
\text { relevantes en la actividad matemática (modelización, } \\
\text { argumentación, resolución de problemas, conexiones, etc.). }\end{array}$ \\
\hline \multirow[t]{4}{*}{ Representatividad } & $\checkmark$ & $\begin{array}{l}\text { Los significados parciales (definiciones, propiedades, } \\
\text { procedimientos, etc.) son una muestra representativa de la } \\
\text { complejidad de la noción matemática que se quiere enseñar }\end{array}$ \\
\hline & $\checkmark$ & $\begin{array}{l}\text { Los significados parciales definiciones, propiedades, } \\
\text { procedimientos, etc.) son una muestra representativa de la } \\
\text { complejidad contemplada en el currículo de la noción matemática } \\
\text { que se quiere enseñar. }\end{array}$ \\
\hline & $\checkmark$ & $\begin{array}{l}\text { Para uno o varios significados parciales seleccionados para su } \\
\text { implementación, ¿se contempla una muestra representativa de } \\
\text { problemas? }\end{array}$ \\
\hline & & $\begin{array}{l}\text { Para uno o varios significados parciales seleccionados para su } \\
\text { implementación, ¿se contempla el uso de diferentes modos de } \\
\text { expresión (verbal, gráfico, simbólico...), tratamientos y conversiones } \\
\text { entre los mismos? }\end{array}$ \\
\hline
\end{tabular}


los resultados de la investigación en el área de Didáctica de las Matemáticas. En particular, para la idoneidad epistémica se ha tenido en cuenta un principio fundamental del EOS que, con los matices propios de cada enfoque, es (o puede ser) asumido por otros enfoques teóricos del área. Nos referimos al principio que se puede formular de la siguiente manera: los objetos matemáticos emergen de las prácticas, lo cual conlleva su complejidad (FONT, GODINO y GALLARDO, 2013; RONDERO y FONT, 2015). De este principio se deriva un componente (representatividad) cuyo objetivo es que se tenga en cuenta, dentro de lo posible, una muestra representativa de dicha complejidad en el diseño y rediseño de las secuencias didácticas para la enseñanza de un determinado objeto matemático (PINO-FAN, CASTRO, GODINO y FONT, 2013).

EL COMPONENTE "MUESTRA REPRESENTATIVA DE LA COMPLEJIDAD DEL OBJETO MATEMÁTICO" Y SU RELACIÓN CON LAS NOCIONES DE "CONEXIÓN" Y DE “CONTEXTO"

En esta sección nos planteamos responder primero a la pregunta ¿cuáles son los diferentes usos que se hacen de los términos "contexto" en la Didáctica de las Matemáticas? para después proponer un punto de vista pragmatista sobre el papel de los contextos intra y extra matemáticos, y de sus conexiones, en la emergencia de los objetos matemáticos en el proceso de su enseñanza y aprendizaje

Una primera observación es que la noción de proceso de conexión y la noción de contexto están estrechamente relacionados. En efecto, los principios y estándares del NCTM (2000) consideran el proceso de conexión, que es entendido como aquel que permite conectar diferentes contenidos matemáticos entre sí y también permite conectar las matemáticas con contextos extra matemáticos.

Con relación al término contexto, hay básicamente dos usos. Uno consiste en considerar el contexto como una situación que cae bajo el dominio de un determinado objeto matemático, mientras que el otro consiste en dar más detalles sobre el entorno. En el primer uso se pone la mirada en la relación entre el ejemplar y el tipo (entre lo concreto y lo abstracto, lo particular y lo general o entre lo extensivo y lo intensivo). En el segundo uso se pone el foco en las notaciones, las propiedades, las definiciones que enmarcan al término matemático que se considera, y se habla, por ejemplo, de contexto algebraico, de contexto geométrico, etc.

Un ejemplo para ilustrar estos dos usos lo tenemos en el caso de la circunferencia. Supongamos que se ha encontrado un trozo de un plato circular de cerámica antiguo y que se quiere exponer en un museo. Para ello, se tiene que construir un soporte circular con las dimensiones reales del plato y encajar, sobre él, el trozo de cerámica encontrado. Este problema se puede resolver dibujando tres puntos del borde del trozo de cerámica que determinan dos segmentos, después se construyen las dos mediatrices y el punto de corte de estas rectas nos da el centro de la circunferencia del plato completo; pero también se puede hacer dibujando primero un sistema de coordenadas, segundo determinado las coordenadas de los tres puntos $\mathrm{y}$, tercero, plantear un sistema de tres ecuaciones lo cual nos permite hallar los tres parámetros de la ecuación de la circunferencia $x^{2}+y^{2}+A x+$ 
$B y+C=0$. En ambos casos el contexto del trozo de cerámica se puede considerar un contexto extra matemático que cae bajo el dominio de la noción de circunferencia, pero, en un caso, la circunferencia se sitúa en un contexto intra matemático de Geometría Elemental y, en el otro, en un contexto intra matemático de Geometría Analítica.

Figura 1 - Contexto extra matemático.
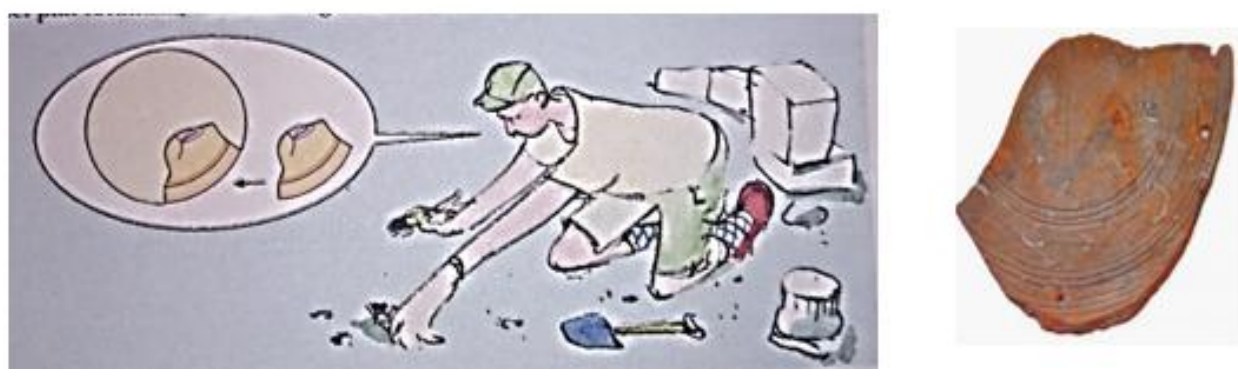

(Fuente: Barceló et. al., 2002, p. 232)

El término contexto extra matemático conlleva pensar en la conexión que se puede dar entre el mundo real y las matemáticas. Para las situaciones extra matemáticas que contextualizan un objeto matemático se han propuesto diferentes nombres y clasificaciones. "Problemas contextualizados", "problemas del mundo real" "problemas relacionados con el trabajo", "problemas situados" son sólo algunos de los diferentes nombres que se da a las tareas escolares que simulan situaciones del mundo real. La conexión entre un objeto matemático y un contexto extra matemático que cae bajo su dominio, nos sitúa en la perspectiva particular-general y conlleva el problema de la trasferencia del conocimiento usado o generado en un contexto a otro contexto diferente y, más en concreto, el problema de la trasferencia del conocimiento aprendido en la escuela a las situaciones prácticas de la vida cotidiana y viceversa. Se plantea, pues, un problema relacionado con la construcción del significado.

Con relación a la construcción del significado en nuestra opinión hay, básicamente, dos posiciones: 1) la semántica o referencial y 2) la pragmatista. El punto de vista semántico considera que el aspecto clave en la construcción del significado es el referente, es decir los ejemplos del objeto matemático. Desde esta perspectiva el problema de la contextualización más o menos sería el siguiente: 1) el profesor tiene que enseñar un objeto matemático descontextualizado y lo que tiene que hacer es buscar ejemplos concretos de dicho objeto, a ser posible situaciones reales, es decir tiene que contextualizar. A partir de estas situaciones, y como resultado del proceso de enseñanza-aprendizaje, el alumno ha de descontextualizar para construir el objeto matemático y posteriormente aplicar este objeto matemático a otros contextos (es decir ha de volver a contextualizar). Desde esta perspectiva todos los ejemplos son iguales ya que se considera que los ejemplos del objeto forman una clase homogénea. Este punto de vista tiene 
dificultades para explicar, entre otras cosas, la falta de transferencia de un contexto a otro y los fenómenos del prototipo - nos referimos a que las personas no suelen considerar los ejemplos de un concepto como una clase homogénea.

El filósofo Frege introdujo la diferencia entre sentido y referente de un término. El referente es el objeto nombrado o al cual se hace referencia, mientras que el sentido es la manera de presentación. El ejemplo de la mediatriz puede ilustrar la diferencia entre sentido y referente. Podemos definir la mediatriz de un segmento como la perpendicular que pasa por el punto medio o como el lugar geométrico formado por todos los puntos equidistantes de los extremos del segmento. En cada definición relacionamos el término mediatriz con otros términos diferentes, pero nos estamos refiriendo al mismo referente. Ahora bien, para muchas personas, las dos definiciones pueden tener sentidos distintos porque la manera de presentación (la conexión con otros términos) permite considerar que cada una de estas definiciones puede tener un referente diferente. Entender que las dos definiciones son equivalentes informa que dos definiciones, el sentido de las cuales se ha fijado de antemano, de manera que pueden referirse a referentes diferentes, se refieren al mismo referente.

Cada definición hay que entenderla como una definición-regla que, de entrada, no parece que indique que haya algo que sea preciso hacer. A las definiciones-reglas cabe atribuir valores veritativos (verdadero y falso). Por ejemplo, si consideramos la definición que dice que la mediatriz de un segmento es la perpendicular que pasa por el punto medio, dada una perpendicular podemos decir si es o no la mediatriz en función de que pase o no por el punto medio. Ahora bien, de una definición-regla se puede deducir una regla práctica (un procedimiento) que nos da instrucciones para construir la mediatriz. Esta práctica se puede dar en diferentes situaciones, por lo que se puede afirmar que una definición y su procedimiento derivado generan un conjunto de prácticas. A su vez, otra definición equivalente generará otro subconjunto de prácticas. Por tanto, en cada definición de mediatriz relacionamos el término definido con términos diferentes y con procedimientos de construcción diferentes. Pero no solo con procedimientos diferentes, también con proposiciones y notaciones diferentes. Dicho de otra manera, tenemos diferentes contextos intra matemáticos.

Desde un punto de vista pragmatista, el significado de un objeto matemático se entiende como el conjunto de prácticas en la que dicho objeto interviene de una manera determinante. Es decir, supone disponer de prácticas con respecto al campo de experiencia que el objeto abarca. Cuando se define el significado de un objeto matemático en términos de prácticas, tal como se propone en el pragmatismo, resulta que el significado de un objeto matemático queda ligado a otros significados y a otros objetos, puesto que en las prácticas interviene dicho objeto conjuntamente con otros objetos matemáticos. Este hecho, permite distinguir dos términos que resultan difíciles de diferenciar, nos referimos a los términos sentido y significado. En efecto, puesto que el objeto se puede relacionar con unos u otros objetos según el contexto, el tipo de notación, etc. para dar lugar a diferentes prácticas, podemos entender el sentido como un significado parcial, esto es como un subconjunto (sentido) del sistema de prácticas en las que el objeto es determinante (significado). puede parcelar en diferentes clases de prácticas más específicas que son utilizadas 
en un determinado contexto y con un determinado tipo de notación produciendo un determinado sentido. Cada contexto ayuda a generar sentido (permite generar un subconjunto de prácticas), pero no genera todos los sentidos.

Un objeto matemático, que se ha originado como un emergente del sistema de prácticas que permite resolver un determinado campo de problemas, con el paso del tiempo queda enmarcado en diferentes programas de investigación. Cada nuevo programa de investigación permite resolver nuevos tipos de problemas, aplicar nuevos procedimientos, relacionar el objeto (y por tanto definir) de una manera diferente, utilizar nuevas representaciones, etc. De esta manera, con el paso del tiempo aparecen nuevos subconjuntos de prácticas (sentidos) que amplían el significado del objeto.

Según Font, Godino y Gallardo (2013), de acuerdo con el punto de vista pragmatista, para analizar un texto matemático y, más en general, la actividad matemática, sea profesional o escolar, es necesario contemplar como mínimo los siguientes elementos: 1) notaciones, representaciones (lenguaje), 2) situacionesproblema 3) definiciones, 4) procedimientos, técnicas, etc. 5) proposiciones, propiedades, teoremas, etc. y 6) argumentos. Estos seis tipos de elementos se articulan formando configuraciones epistémicas y se pueden entender como un contexto intra matemático. Se trata de una herramienta que puede ser útil para describir la complejidad de los objetos matemáticos y de las prácticas de las cuales emergen. En el EOS, la introducción de la dualidad unitaria-sistémica permite reformular la visión "ingenua" de que "hay un mismo objeto matemático (mediatriz, para seguir con el ejemplo) con distintas representaciones". Lo que hay es un sistema complejo de prácticas, que permiten resolver problemas, en las que el objeto matemático "mediatriz" no aparece directamente, lo que si aparece son representaciones de la mediatriz, diferentes definiciones de la mediatriz, proposiciones y propiedades de la mediatriz, procedimientos y técnicas que se aplican a la mediatriz y argumentos sobre la mediatriz. Dicho de otra manera, a lo largo de la historia se han ido generando diferentes configuraciones epistémicas para el estudio de la mediatriz, algunas de las cuales han servido para generalizar a las preexistentes

Desde esta perspectiva, un criterio de idoneidad de una trayectoria didáctica de enseñanza y aprendizaje para un objeto matemático es que el conjunto de prácticas implementadas sea un conjunto lo más representativo posible del sistema de prácticas que son el significado del objeto. Dicho en términos de contextos, hay que presentar a los alumnos una muestra de contextos intra matemáticos representativa, una muestra de contextos que permita construir una muestra representativa de los diferentes sentidos del objeto. Por otra parte, una vez seleccionada una muestra representativa de contextos intra matemáticos hay que seleccionar los contextos extra matemáticos que permiten hacer emerger las configuraciones epistémicas en las que se concretan dichos contextos intra matemáticos.

La mirada compleja aplicada al objeto matemático permite profundizar en el proceso de conexión entre significados parciales de un mismo objeto. Por una parte, la complejidad, estructurada en términos de un conjunto de configuraciones epistémicas, precisa cuáles son los componentes a conectar. Por otra parte, cada una de estas configuraciones epistémicas, entendidas como contextos intra 
matemáticos, se tienen que relacionar con los contextos extra matemáticos mediante la relación particular-general.

\section{UN EJEMPLO: LA ENSEÑANZA DEL TEOREMA DE TALES EN LA EDUCACIÓN BÁSICA}

Vamos a concretar las reflexiones de los apartados anteriores para el caso de la enseñanza del Teorema de Tales en la educación básica. En las orientaciones curriculares de esta etapa se pretende que el alumno aplique el teorema de Tales a diferentes contextos extra matemáticos. Por ejemplo, se trata que, como resultado del proceso de enseñanza y aprendizaje, el alumno pueda resolver estos dos problemas contextualizados, adaptados de Fargas y Font (1997):

$\checkmark$ Tarea 1: la tarea representa dos edificios de una calle que celebra las fiestas del barrio y un cable con elementos decorativos ( $A, B, C$ y $D)$ de tipo festivo que va de un edificio a otro. Las flechas representan los rayos del sol, $C^{\prime}$ es la sombra de C y D' la de D. Sabiendo que AB mide 1,5 metros, que CD mide 0,7 metros y que $C^{\prime} D^{\prime}$ mide 0,6 metros, calcula la longitud del segmento de sombra $A^{\prime} B^{\prime}$.

Figura 2 - Ilustración de la tarea 1.

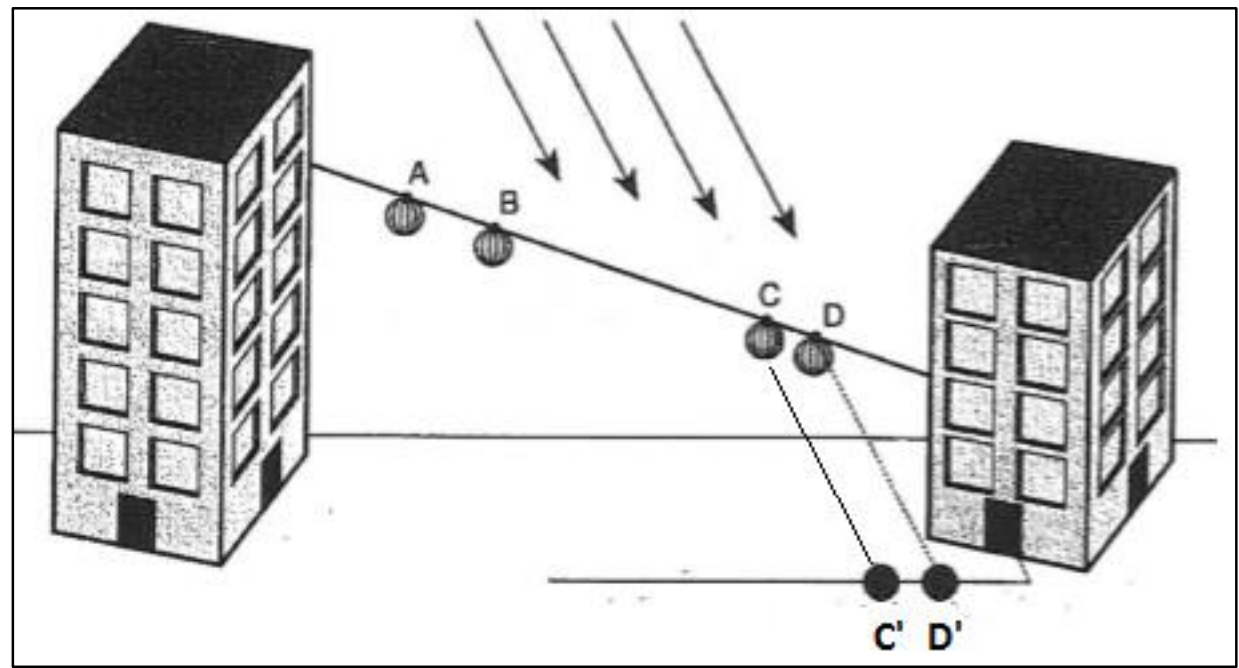

(Fuente: una adaptación de Fargas y Font, 1997, p.25)

$\checkmark$ Tarea 2: Una subestación eléctrica tiene que suministrar corriente eléctrica a una zona deportiva. Entre ambas se halla un lago que dificulta la medición de la distancia que las separa. Calcula la distancia que separa la subestación eléctrica de la zona deportiva y explica los instrumentos que utilizarías para realizar esta medición en la práctica.

Figura 3 - Ilustración de la tarea 2. 


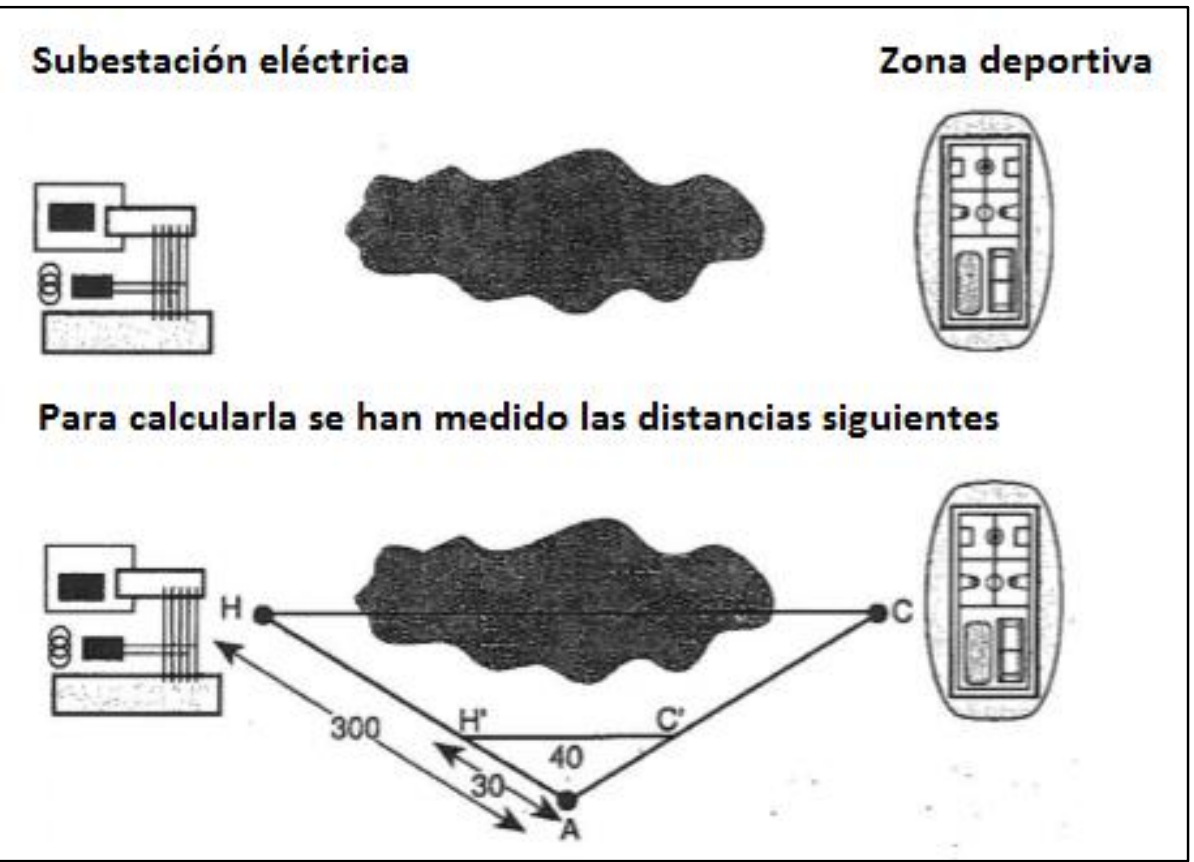

(Fuente: Fargas y Font, 1997, p. 31)

Se trata de dos tareas que necesitan diferentes formulaciones del teorema de Tales. Para la primera tarea necesitamos la siguiente versión del teorema de Tales (que llamaremos extendida): Los segmentos determinados por rectas paralelas son proporcionales $\frac{A B}{C D}=\frac{A^{\prime} B^{\prime}}{C^{\prime} D^{\prime}}$

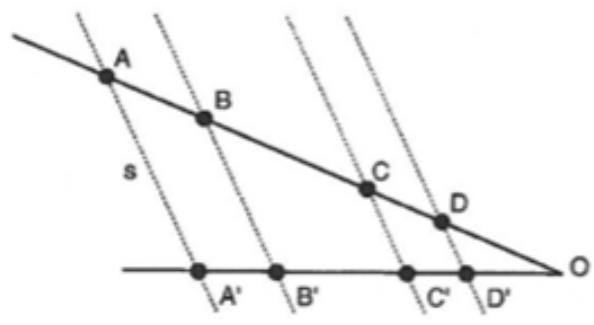

Figura 4 - Teorema de Tales (extendido).

Figura 45- Teorema de Tales (reducido). 
Las secuencias de tareas que pretenden enseñar el teorema de Tales en la secundaria en España suelen hacerlo de dos maneras diferentes. En un caso, se parte de la idea de figuras semejantes y se llega a la noción de triángulos en posición de tales y se enuncia el teorema de Tales en la versión que hemos llamado reducida. La configuración epistémica (que llamaremos configuración epistémica CE1 - triángulos semejantes) que describe esta secuencia de tareas sería la siguiente:

Tabla 2 - Configuración epistémica de los triángulos semejantes (CE1).

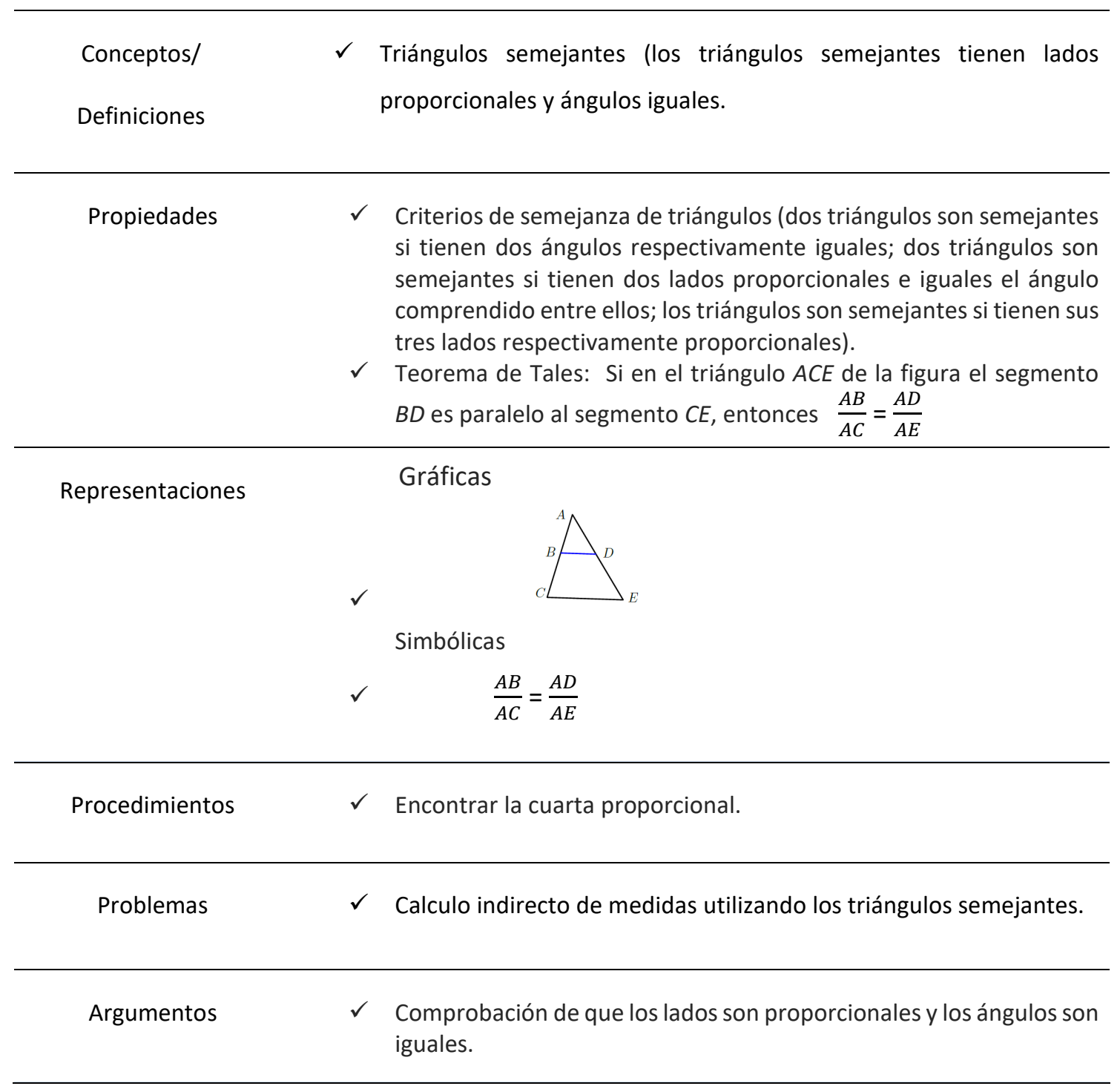


epistémica (que llamaremos configuración epistémica CE2-Teorema de Tales extendido)

Tabla 3 - Configuración epistémica del Teorema de Tales extendido (CE2).

\begin{tabular}{|c|c|c|}
\hline $\begin{array}{l}\text { Conceptos/ } \\
\text { Definiciones }\end{array}$ & $\checkmark$ & $\begin{array}{l}\text { Proyección de un punto, proyección de un segmento, proporción, } \\
\text { triángulos en posición de tales. }\end{array}$ \\
\hline \multirow[t]{2}{*}{ Propiedades } & $\checkmark$ & $\begin{array}{l}\text { P1: Los segmentos determinados por rectas paralelas son } \\
\text { proporcionales. }\end{array}$ \\
\hline & $\checkmark$ & $\begin{array}{l}\text { P2: Dos triángulos en posición de tales tienen los lados } \\
\text { proporcionales y los lados iguales }\end{array}$ \\
\hline Procedimientos & $\checkmark$ & Encontrar la cuarta proporcional. \\
\hline \multirow[t]{3}{*}{ Representaciones } & & Gráficas \\
\hline & & Simbólicas \\
\hline & & $\frac{A B}{C D}=\frac{A^{\prime} B^{\prime}}{C^{\prime} D^{\prime}}$ \\
\hline \multirow[t]{2}{*}{ Problemas } & $\checkmark$ & $\begin{array}{l}\text { Calculo indirecto de medidas utilizando los triángulos en posición de } \\
\text { tales. }\end{array}$ \\
\hline & $\checkmark$ & $\begin{array}{l}\text { Hallar uno de los } 4 \text { segmentos determinados por rectas paralelas } \\
\text { sobre dos rectas secantes }\end{array}$ \\
\hline \multirow[t]{2}{*}{ Argumentos } & $\checkmark$ & Comprobación del teorema de Tales. \\
\hline & $\checkmark$ & $\begin{array}{l}\text { Demostración de que los triángulos en posición de tales tienen los } \\
\text { lados proporcionales y los ángulos iguales (aplicando dos veces el } \\
\text { teorema de tales) }\end{array}$ \\
\hline
\end{tabular}


llamado triángulos semejantes. En un primer momento se puede explicar la CE1, pero en algún otro momento es necesario explicar la CE2 y conectarla con la CE1, por ejemplo, haciendo ver que la aplicación repetida del teorema de Tales permite demostrar que los triángulos en posición de Tales tienen los lados proporcionales.

\section{LA REFLEXIÓN SOBRE LA COMPLEJIDAD DEL OBJETO MATEMÁTICO EN LA FORMACIÓN INICIAL DEL PROFESORADO.}

En diferentes procesos de formación de profesores de matemáticas de España, México, Brasil, Ecuador, Chile, Venezuela y Argentina (RAMOS, 2006; RAMOS y FONT, 2008; GIMÉNEZ, VANEGAS, FONT y FERRERES, 2012; GIMÉNEZ, FONT y VANEGAS, 2013; FERRERES y VANEGAS, 2015; POCHULU, FONT y RODRÍGUEZ, 2016; BREDA, 2016; BREDA y LIMA, 2016; BREDA, FONT y LIMA, 2016; BREDA, PINO-FAN y FONT, 2016; PINO-FAN, GODINO y FONT, 2016; SECKEL, 2016; BREDA, PINO-FAN \& FONT, 2017) se han realizado un conjunto de investigaciones que tienen como finalidad investigar el uso del constructo criterios de idoneidad didáctica, propuestos por el Enfoque Ontosemiótico de la Cognición e Instrucción Matemática (GODINO, BATANERO y FONT, 2007; BREDA, FONT y LIMA, 2015), como herramienta para organizar la reflexión del profesor sobre su práctica, cuando esta reflexión está orientada hacia la valoración y mejora de la práctica. Uno de los espacios de formación investigados en esta amplia investigación es el Trabajo de Fin de Máster (TFM).

Estas investigaciones han puesto de manifiesto diferentes aspectos, de los cuales queremos señalar aquí los siguientes:

1) Aunque no se incorpore explícitamente en el dispositivo formativo de los profesores, la enseñanza de los componentes y descriptores de los criterios de idoneidad didáctica, algunos de ellos, y en particular el componente <<muestra representativa de la complejidad del objeto matemático〉>, están presentes de manera implícita cuando los profesores o futuros profesores hacen valoraciones de propuestas didácticas (suyas o de otros).

En Breda (2016), Breda y Lima (2016), Breda, Pino-Fan y Font (2017) se muestra que, en muchos casos, los profesores, sin haber recibido una instrucción sobre el uso de los criterios de idoneidad, tienen en cuenta de manera implícita este descriptor (conjuntamente con otros) cuando tiene que justificar que su propuesta didáctica representa una innovación.

Un ejemplo de dicha evidencia se encuentra en el análisis didáctico realizado por profesores que cursan el Mestrado Profissional em Matemática em Rede Nacional (PROFMAT) cuándo tienen que justificar que sus propuestas didácticas (descriptas en el trabajo de conclusión de curso) son innovadoras y representan una mejora con relación a la enseñanza de las matemáticas que se realiza habitualmente, (BREDA, 2016).

Para ilustrar este tipo de análisis didáctico que hacen los profesores, en Breda y Lima (2016) se presenta un caso que trata de la implementación de una secuencia didáctica cuya propuesta innovadora consiste en implementar el estudio de los polinomios por medio de un abordaje funcional. una breve revisión histórica sobre los polinomios destacando los principales 
resultados y personajes que contribuyeron a su estudio. En un según momento el autor trata sobre la forma en que los polinomios se presentan en los libros de la etapa de Enseñanza Secundaria del Brasil. Presenta una reflexión sobre los Parámetros Curriculares Nacionales del Brasil para la Etapa Básica (Primaria y Secundaria) (PCN) destacando que hay un eje dedicado al álgebra que remarca la conexión de ésta con las funciones.

En un tercer momento, el autor se propone identificar los polinomios como funciones polinómicas y, después, introducir los conceptos de valor numérico y de raíz de un polinomio desde un punto de vista funcional. Utilizando el GeoGebra presenta un estudio de las funciones polinómicas, mostrando la relación que hay entre el número de veces que el gráfico corta al eje $x$ con el grado del polinomio. Seguidamente, el autor se preocupa en dar énfasis al número de operaciones necesarias para el cálculo del valor numérico y en buscar una fórmula que nos dé el número de estas operaciones. A continuación, se dedica a presentar el método de Ruffini y también se preocupa por el número de operaciones necesarias para encontrar el valor numérico de una función polinómica por este método. Luego muestra que el método de Ruffini disminuye el número de operaciones necesario para calcular el valor numérico. En lo que sigue, el autor se preocupa del cálculo de las raíces de un polinomio mediante el Teorema del Valor Intermedio y en justificar el Teorema Fundamental del Álgebra de manera gráfica, relacionando el gráfico generado por el polinomio con los puntos de corte del gráfico con el eje de las abscisas. También introduce la Regla del Signo de Descartes para contar las raíces positivas y negativas de un polinomio y, por último, explica el Teorema de las Raíces Racionales para determinar las raíces racionales de un polinomio.

En un cuarto momento el autor muestra la implementación de parte de la propuesta planificada en el capítulo tres, en el tercer año de Enseñanza Secundaria, conectando, mediante la definición de polinomio, la idea de valor numérico y la de raíz de un polinomio desde un punto de vista funcional. Seguidamente realiza actividades que tienen como objetivo encontrar las raíces de polinomios a través del Teorema del Valor Intermedio y de la construcción del gráfico del polinomio con el GeoGebra. A continuación, realiza una actividad que consiste en que los alumnos hallen las raíces del polinomio cuando este está expresado en forma factorial. El autor se preocupa por hacer que los alumnos dividan un polinomio por uno de sus factores sin explicar antes un método de división, o sea, los alumnos deben multiplicar los factores excluyendo aquel que se sugiere como divisor, proponiendo, a partir de esto, una actividad para introducir la regla de Ruffini para la división de polinomios. Además de esto, utiliza la regla de Ruffini para calcular los valores numéricos y enfatiza que el cálculo del valor numérico por el método de Ruffini permite menos operaciones que cuando se usa o método de sustituir en la expresión del polinomio. Finaliza la actividad con la introducción del esbozo del gráfico en el GeoGebra para que los alumnos visualicen las raíces y las concavidades.

Al final el autor argumenta que su propuesta de presentar los polinomios de forma práctica, intuitiva y con recursos computacionales es innovadora de acuerdo con los objetivos del PROFMAT. También comenta que antes de la experimentación tenía dudas sobre si su propuesta se podría aplicar por cuestiones de tiempo y si 
universidad. Comenta, también, que después de la aplicación de la propuesta los alumnos no presentaron dificultades para resolver las tareas, que el tiempo fue suficiente y que los alumnos estaban más entusiasmados, concluyendo que su propuesta es viable y que se adapta a las directrices curriculares nacionales.

Presentada una síntesis de la propuesta didáctica de Dierings (2014), a continuación se muestran algunas evidencias de las reflexiones implícitas que el autor hace sobre la complejidad de los polinomios, haciendo observar que estos pueden ser presentados en un contexto algebraico o en un contexto funcional y muestra una secuencia de actividades que permiten el establecimiento de una conexión entre esos dos contextos intra-matemáticos. De hecho, el autor, asume la centralidad del estudio de los polinomios por medio de un abordaje funcional:

Generalmente, cuando empezamos a desarrollar este contenido en el tercer año nos surge la siguiente duda: ¿Es necesario distinguir entre polinomio y función polinómica? Según Lima (2006) no es necesaria esta distinción. "Tenga en cuenta que el concepto de polinomio contempla apenas la lista de sus coeficientes y la manera en la que los sumamos y multiplicamos; al referirse a la función polinómica, empezamos a estar interesados en la correspondencia entre números complejos establecida por el valor que la función toma en cada punto. Es claro que cada polinomio representa una única función polinómica. Por otra parte, hemos visto anteriormente que dos funciones polinómicas sólo son iguales cuando tienen la misma lista de coeficientes. En otras palabras, dos funciones polinómicas solo son iguales cuando los polinomios asociados a ellas son iguales. Por lo tanto, la función polinómica también corresponde a un único polinomio. De esta manera, hay una correspondencia biunívoca entre las funciones polinómicas y polinomios, lo que nos permite, sin riesgo de confusión, hacer referencia indistintamente al polinomio $p$ o la función polinómica $p$. A menudo es conveniente referirse a un "polinomio $p(x)$ ", especialmente en situaciones donde otros polinomios aparezcan descritos sólo por su expresión. (DIERINGS, 2014, p.22)

(...) no es difícil para el estudiante entender que a cada función polinómica puede asociarse a una gráfica representada en el plano cartesiano. Así, presentamos algunas ideas interesantes, como la idea de que, en el caso de la función, para cada valor real de $x$ tenemos un valor numérico correspondiente, la noción de continuidad del polinomio, la intersección de la gráfica de la función con los ejes cartesianos, el signo del polinomio, el crecimiento y decrecimiento. Llamamos la atención sobre el crecimiento y el decrecimiento, en el sentido de visualizar los puntos máximos y mínimos, así como la constatación de que los valores numéricos pueden ser los mismos para diferentes valores de $x$. (DIERINGS, 2014, p. 23)

Vemos pues que el autor explica, en su propuesta, que planea realizar una conexión intra- matemática y se preocupa por conectar el tratamiento algebraico de los polinomios con el funcional.

2) Incorporar el componente <<muestra representativa de la complejidad del objeto matemático >> para valorar la idoneidad epistémica de un proceso de enseñanza y aprendizaje no es tarea fácil, ni para los formadores ni para sus alumnos (futuros profesores), pero se puede enseñar como parte del proceso de formación del profesorado. A continuación, ponemos dos ejemplos para ilustrar algunos momentos del proceso de enseñanza y aprendizaje de este componente realizado en dos dispositivos de formación inicial de profesores de matemáticas en España y Chile. 
El primer ejemplo presentar el uso del componente $<<$ muestra representativa de la complejidad del objeto matemático > en el proceso de reflexión (explicado en su Trabajo Fin de Máster) de una futura profesora (RUIZ, 2014), con el fin de mejorar la implementación de una unidad didáctica sobre la función de segundo grado en el cuarto año de la Enseñanza Secundaria Obligatoria (ESO) de España (alumnos de 16 años). El uso de los criterios de idoneidad didáctica fue enseñado en el proceso formativo de esta futura profesora como una herramienta metodológica para promover y apoyar la reflexión sobre su práctica.

En las orientaciones generales del Trabajo Fin de Máster que se dan a los alumnos, en el caso del Máster de Formación de Profesorado de Secundaria que ha cursado la futura profesora Ruiz, se dice que debe ser un trabajo que debe contribuir a reflexionar y profundizar en el análisis de su propia práctica, posibilitando proponer elementos de mejora de la misma. El TFM es la culminación de un ciclo formativo en el que, primero, el alumno ha planificado e implementado una unidad didáctica en el periodo de prácticas. Para después (en el TFM) realizar el análisis y valoración de la idoneidad de la unidad didáctica implementada y formular una propuesta de mejora justificada de dicha unidad didáctica. Para la parte final de este ciclo, se sugiere a los futuros docentes que en su análisis consideren responder a preguntas como las siguientes: a) ¿He enseñado unas matemáticas de calidad? ¿Se puede mejorar esta calidad? ¿Cómo? b) Los alumnos podían aprender con las actividades propuestas? ¿Han aprendido? ¿Por qué no? c) ¿Se podría mejorar la gestión de la clase? d) ¿Usé los recursos adecuados? ¿El tiempo estuvo bien gestionado? e) ¿Cómo se ha considerado una perspectiva ecológica en las condiciones generales del trabajo? Para responder a estas preguntas en las diferentes asignaturas que intervienen en el ciclo formativo se presentan elementos de valoración de la calidad de los procesos de estudio, en concreto los criterios de idoneidad didáctica propuestos por el EOS (GODINO, BATANERO y FONT, 2007), así como la pauta de componentes y descriptores de dichos criterios que permite aplicarlos (BREDA y LIMA, 2016).

La futura profesora Ruiz (2014), en su TFM presenta la valoración y el rediseño de una propuesta didáctica sobre la función de segundo grado, para un grupo de alumnos del cuarto año de la Enseñanza Secundaria Obligatoria (ESO) de spaña (15-16 años de edad). Este TFM está organizado en cinco capítulos; en el primero se realiza una introducción al TFM, en el segundo se presenta un resumen de la implementación de la unidad didáctica (la explicación detallada se halla en su memoria de prácticas). En el tercer capítulo se explica la valoración de los seis criterios de idoneidad didáctica. En el cuarto capítulo se especifican los aspectos que se proponen mejorar en una futura implementación y se detalla el rediseño de las tareas para ello. El último capítulo termina con unas consideraciones finales sobre el TFM y sobre su experiencia en el máster.

En el tercer capítulo, donde realiza la valoración de los seis criterios de idoneidad didáctica, cuando valora la idoneidad epistémica, con relación al componente $<<$ muestra representativa de la complejidad del objeto matemático $>>$ dice que éste se debe mejorar en el rediseño de la unidad didáctica de cara a futuras implementaciones y argumenta su valoración negativa de dicho componente de la manera siguiente: 
representativa de la complejidad de la función cuadrática. Han faltado significados parciales y sus conexiones. (RUIZ, 2014, p. 35-36).

La muestra de actividades presentadas en la UD no ha sido representativa. Han faltado problemas de contextos significativos de la aplicación de la parábola, como pueden ser de tiro parabólico, de fuentes (por ejemplo, Montjuïc), de luz de faros, etc. (RUIZ, 2014, p. 35-36).

El segundo ejemplo que explicamos brevemente es cómo se usó el componente $<$ <muestra representativa de la complejidad del objeto matemático > en un dispositivo de formación implementado en Chile. En Seckel (2016) se describe un dispositivo de formación de una formadora de profesores de educación básica con mención en matemática cuyo objetivo es desarrollar la competencia reflexiva de esta profesora y el de sus alumnos.

Un aspecto que se trató en el diseño e implementación del ciclo formativo que recibió esta profesora fue cómo abordar el componente "muestra representativa de la complejidad del objeto matemático (proporcionalidad en este caso). La forma como decidió enfocarlo la profesora fue mediante el diseño de un mapa conceptual de la complejidad matemática de la proporcionalidad (figura 5) que ella explicaría a sus alumnos.

Los investigadores, después de analizar el mapa conceptual sobre la proporcionalidad confeccionado por la profesora, realizaron una retroalimentación a la profesora, haciéndole las siguientes observaciones sobre la presencia los diferentes significados parciales que se podían inferir del mapa:

Dado que en el mapa hay una referencia a Euclides, podemos inferir que se tiene en mente el significado de las proporciones en las matemáticas griegas, donde la proporción era interpretada como la expresión: $a: b:: c: d$. Desde este enfoque, la proporcionalidad tenía personalidad propia, prácticamente desvinculada de las fracciones y con un álgebra que se fundaba sobre reglas propias de la proporcionalidad.

El segundo significado que se infiere en el mapa tiene relación con las proporciones desde un punto de vista algébrico. A partir del desarrollo del álgebra se llega a la identificación de una razón con una fracción, perdiéndose buena parte de la identidad propia de la proporcionalidad. La proporción $a: b$ :: $c: d$ pasa a ser interpretada como una igualdad entre fracciones equivalentes.

El tercer significado tiene relación con las proporciones desde el punto de vista funcional. A partir del estudio de la relación entre magnitudes se inicia una nueva interpretación de la proporcionalidad, que es entendida, primero, como una relación entre magnitudes (proporcionales y no proporcionales) y, después, como una relación entre variables. En el mapa dicho significado parcial se observa en dos partes del mapa, en el rectángulo dedicado a las magnitudes y en la columna dedicada a las funciones.

El cuarto significado tiene relación con las proporciones desde un punto de vista geométrico, donde se estudia la proporcionalidad entre segmentos y los criterios de semejanzas entre figuras, en particular, de los triángulos, que aparecen sobre todo en la columna de razones. 
Figura 5 - Mapa conceptual de la complejidad de la proporcionalidad 


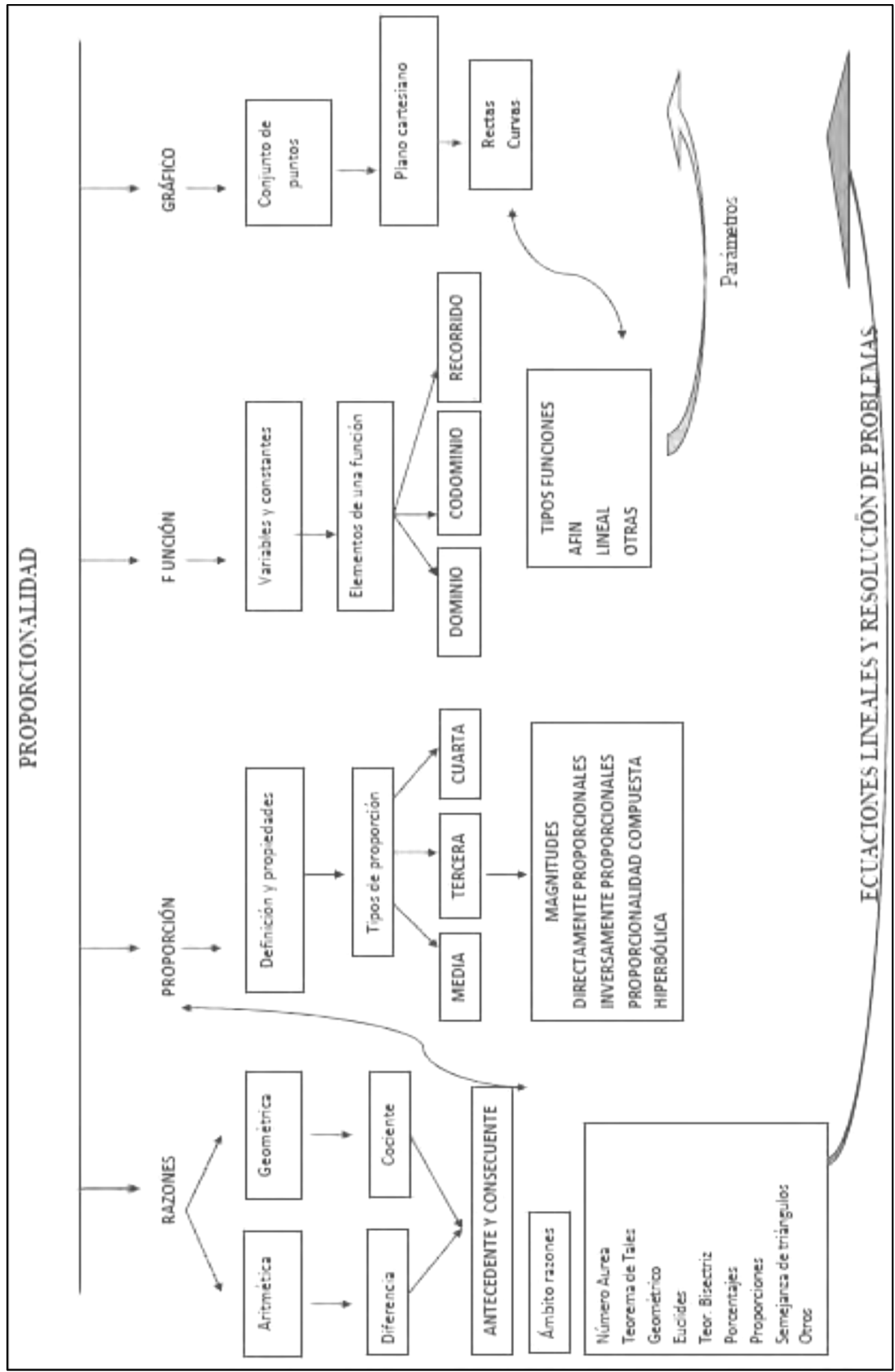

Fuente: (Seckel, 2016, p. 195)

De alguna manera se puede decir que en la confección del mapa se han tenido en cuenta estos significados, sin embargo, quedan ocultos otros aspectos, como son:

$\checkmark$ El papel importante de las fracciones. En el significado algébrico las razones son interpretadas como fracciones y la proporción se interpreta como una relación entre fracciones equivalentes. 
$\checkmark$ No aparecen procedimientos (por ejemplo: regla de tres o producto en cruzado).

$\checkmark \quad$ No se habla de tipos de problemas (por ejemplo: problemas de valor faltante, de comparación o problemas cualitativos de predicción o de comparación).

Los comentarios anteriores permitieron un feedback a la profesora que le ayudó a profundizar en el uso del descriptor $<<$ muestra representativa de la complejidad del objeto matemático > en sus clases futuras.

\section{CONSIDERACIONES FINALES}

En este artículo se muestra como la tendencia a considerar que las matemáticas se deben aplicar a contextos extra matemáticos conlleva la reflexión sobre la complejidad de los objetos matemáticos. Por otra parte, las partes de esta complejidad se han de conectar entre ellas y con situaciones extra matemáticas, lo cual nos lleva a relacionar la complejidad del objeto matemático con el proceso de conexión.

Se trata de pasar de un punto de vista ingenuo y optimista, qué presupone que el alumno fácilmente realizará la transferencia del conocimiento matemático generado en un solo contexto a otros contextos nuevos y diferentes, a otro punto de vista más prudente en el que, si bien se considera que la posibilidad de transferencia creativa se puede dar, se asume que, sin un trabajo sobre una muestra representativa de la complejidad del objeto matemático que se quiere enseñar y la articulación y conexión de los componentes de dicha complejidad, es difícil que se pueda aplicar el objeto matemático a diferentes contextos.

Por tanto, si queremos que nuestros alumnos apliquen las matemáticas a contextos extra matemáticos y hagan conexiones entre diferentes contenidos matemáticos, como profesores debemos reflexionar sobre la complejidad de los objetos matemáticos y sobre la articulación de las partes en las que estalla dicha complejidad (RONDERO y FONT, 2015). Esta última reflexión nos lleva a la problemática de cómo diseñar dispositivos de formación inicial o continua de profesores para poder formarlos en la reflexión sobre la complejidad del objeto matemático y sus implicaciones para su enseñanza y aprendizaje.

\section{RECONOCIMIENTO}

Trabajo realizado en el marco de los proyectos de investigación EDU2015-64646P, (MINECO/FEDER, UE), REDICE16-1520 (ICE-UB) 


\title{
Some didactic implications derived from the complexity of mathematical objects when these applying to different contexts
}

\begin{abstract}
This work is, fundamentally, a theoretical reflection. In the first part explains the notion of didactic suitability and, in particular, the criterion of epistemic suitability, one of its indicators being the "representative sample of the complexity of the mathematical object". The second part justifies the presence of this indicator from a reflection, made from a pragmatist perspective, on its relationship with the notion of connection process and context. It concludes with a reflection on the use of this descriptor in the training of mathematics teachers.
\end{abstract}

KEY WORDS: Epistemic suitability. Connection. Context. Training of mathematics teachers. 
BARCELÓ, R.; BUJOSA, J. M.; CAÑADILLA, J. L.; FARGAS, M.; FONT, V.

Matemáticas I. Barcelona, España: Almadraba, 2002.

BREDA, A. Melhorias no ensino de matemática na concepção de professores que realizam o mestrado PROFMAT no Rio Grande do Sul: uma análise dos trabalhos de conclusão de curso. 2016. 326f. Tesis (Doctorado en Educación en Ciencias y Matemáticas) - Pontifícia Universidade Católica do Rio Grande do Sul, Porto Alegre, 2016.

BREDA, A.; FONT, V.; LIMA, V. M. R. A noção de idoneidade didática e seu uso na formação de professores de matemática. Jornal Internacional de Estudos em Educação Matemática, v. 8, p. 4-41, 2015.

BREDA, A.; FONT, V. y LIMA, Valderez M. R. Análise das Propostas de Inovação nos Trabalhos de Conclusão de Curso de um Programa de Mestrado Profissional. AIEM. Avances de Investigación en Educación Matemática, v. 10, n. 02, p. 53-72, 2016

BREDA, A.; LIMA, V. M. R. Estudio de caso sobre el análisis didáctico realizado en un trabajo final de un máster para profesores de matemáticas en servicio.

REDIMAT - Journal of Research in Mathematics Education, v. 5, n. 1, p. 74-103, 2016.

BREDA, A.; PINO-FAN, L.; \& FONT, V. Establishing criteria for teachers' reflection on their own practices. In CSÍKOS, C., RAUSCH, A., \& SZITÁNYI, J. (Eds.), Proceedings of the 40th Conference of the International Group for the Psychology of Mathematics Education (Vol. 1, pp. 283). Szeged, Hungary: PME, 2016.

BREDA, A.; PINO-FAN, L. \& FONT, V. Meta didactic-mathematical knowledge of teachers: criteria for the reflection and assessment on teaching practice.

EURASIA Journal of Mathematics, Science and Technology Education, v. 13, n. 6, p.1893-1918, 2017.

DIERINGS, A. R. Ensino de polinômios no Ensino Médio - uma nova abordagem. 2014. 70f. Dissertação (mestrado) - Mestrado Profissional em Matemática em Rede Nacional - PROFMAT, Centro de Ciências Exatas, Universidade Federal de Santa Maria, Santa Maria, 2014.

FARGAS, M. y FONT, V. Trigonometria. Barcelona, España: Castellnou, 1997. 
FERRERES, S y VANEGAS, Y. Uso de criterios de calidad en la reflexión sobre la práctica de los futuros profesores de secundaria de matemáticas. Procedia, v. 196, p. 219-225, 2015.

FONT, V., GODINO, J. D. y GALLARDO, J. The emergence of objects from mathematical practices. Educational Studies in Mathematics, v. 82, p. 97-124, 2013.

FONT, V., PLANAS, N. y GODINO, J. D. Modelo para el análisis didáctico en educación matemática. Infancia y Aprendizaje, v. 33, n. 1, p. 89-105, 2010.

GIMÉNEZ, J.; FONT, V. \& VANEGAS, Y. Designing Professional Tasks for Didactical Analysis as a research process. In: MARGOLINAS, C. (Ed.), Task Design in Mathematics Education. Proceedings of ICMI Study 22: Oxford, 2013.

GIMÉNEZ, J.; VANEGAS, Y.; FONT, V. y FERRERES, S. El papel del trabajo final de Máster en la formación del profesorado de Matemáticas. UNO. Revista de Didáctica de las Matemáticas, 61, 76-86, 2012.

GODINO, J. D.; BATANERO, C. \& FONT, V. The onto-semiotic approach to research in mathematics education. ZDM. The International Journal on Mathematics Education, v. 39, n. 1, p. 127-135, 2007.

GODINO, J. D., BATANERO, C. y FONT, V. Um enfoque onto-semiótico do conhecimento e a instrução matemática. Acta Scientiae. Revista de Ensino de Ciências e Matemática, v. 10, p. 7-37, 2008.

NATIONAL COUNCIL OF TEACHERS OF MATHEMATICS. Principles and Standards for School Mathematics. Reston, VA: NCTM, 2000.

PINO-FAN, L.; CASTRO, W. F.; GODINO, J. D.; FONT, V. Idoneidad epistémica del significado de la derivada en el currículo de bachillerato. PARADIGMA, v. 34, n. 2, p. $123-150,2013$.

PINO-FAN, L., GODINO, J. D., \& FONT, V. Assessing key epistemic features of didactic-mathematical knowledge of prospective teachers: the case of the derivative. Journal of Mathematics Teacher Education, 2016. The final publication is available at Springer via http://dx.doi.org/10.1007/s10857-0169349-8 
POCHULU, M.; FONT, V.; RODRÍGUEZ, M. Desarrollo de la competencia en análisis didáctico de formadores de futuros profesores de matemática a través del diseño de tareas. Revista Latinoamericana de Investigación en Matemática EducativaRELIME, v. 19, n. 01, p. 71-98, 2016.

RAMOS, A. B. Objetos personales, matemáticos y didácticos, del profesorado y cambios institucionales. El caso de la contextualización de las funciones en una Facultad de Ciencias Económicas y Sociales. Tesis doctoral no publicada. Universitat de Barcelona, 2006.

RAMOS, A. B e FONT, V. Criterios de idoneidad y valoración de cambios en el proceso de instrucción matemática. Revista Latinoamericana de Investigación en Matemática Educativa-RELIME, v. 11, n. 2, p. 233-265, 2008.

RONDERO, C. y FONT, V. Articulación de la complejidad matemática de la media aritmética. Enseñanza de las Ciencias, v. 33, n. 2, p. 29-49, 2015.

RUIZ, E. Funcions quadràtiques. 2014.143f. Dissertação (mestrado) - Máster de Formación del Profesorado de Matemáticas de Secundaria, Universitat de Barcelona, 2014.

SECKEL, M. J. Competencia en análisis didáctico en la formación inicial de profesores de educación básica con mención en matemática. 2016. 291f. Tesis (Doctorado en Didàctica de les Ciències Experimentals i la Matemàtica) Universitat de Barcelona, Barcelona, 2016.

Recebido: 2017-06-06

Aprovado: 2017-06-06

DOI: $10.3895 /$ rbect.v10n2.598

Como citar:

FONT, V.; BREDA, A.; SECKEL, M. J. Algunas implicaciones didácticas derivadas de la complejidad de los objetos

matemáticos cuando estos se aplican a distintos contextos. Revista Brasileira de Ensino de Ciência e Tecnologia, v. 10,

n. 2, 2017. Disponível em: <https://revistas.utfpr.edu.br/rbect/article/view/5981>. Acesso em: xxx.

Correspondência: Vicenç Font

Passeig de la Vall d'Hebrón, 171, 08035. Barcelona, España.

Direito autoral: Este artigo está licenciado sob os termos da Licença Creative Commons-Atribuição 4.0

Internacional.

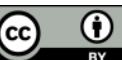

